\title{
ON PROPERTIES OF SEMIPOSITIVE CONES AND SIMPLICIAL CONES*
}

\author{
ARITRA NARAYAN HISABIA ${ }^{\dagger}$ AND MANIDEEPA SAHA ${ }^{\dagger}$
}

\begin{abstract}
For a given nonsingular $n \times n$ matrix $A$, the cone $S_{A}=\{x: A x \geq 0\}$, and its subcone $K_{A}$ lying on the positive orthant, called as semipositive cone, are considered. If the interior of the semipositive cone $K_{A}$ is not empty, then $A$ is named as semipositive matrix. It is known that $K_{A}$ is a proper polyhedral cone. In this paper, it is proved that $S_{A}$ is a simplicial cone and properties of its extremals are analyzed. An one-one relation between simplicial cones and invertible matrices is established. For a proper cone $K$ in $\mathbb{R}^{n}, \pi(K)$ denotes the collection of $n \times n$ matrices that leave $K$ invariant. For a given minimally semipositive matrix (no column-deleted submatrix is semipositive) $A$, it is shown that the invariant cone $\pi\left(K_{A}\right)$ is a simplicial cone.
\end{abstract}

Key words. Extremal, Proper cone, Polyhedral cone, Simplicial cone, Semipositive matrix, Minimally semipositive matrix.

AMS subject classifications. 15A09, 15B48.

1. Introduction. An $m \times n$ real matrix $A$ is called a semipositive (SP) matrix if there exists a positive vector $x$ such that $A x$ is also a positive vector, that is, there exists a positive vector which is mapped to a positive vector under $A$. Non-negative matrices, $M$-matrices, $P$-matrices, and positive definite matrices are some important classes of semipositive matrices. SP matrices first appeared in literature as $S$-matrices named after Stiemke, due to Fiedler and Pták [5]. Motivated by the role of SP matrices in a variety of theoretical and practical problems, mainly in linear complementarity problems, an extensive research is carried in two directions. One is the algebraic properties and the other is cone theoretic properties of SP matrices, which can be reviewed in $[3,4,7,8,11,13,15,17]$.

Let $\mathbb{R}^{m \times n}$ denote the all $m \times n$ real matrices. For simplicity, we write $\mathbb{R}^{n}$ for $\mathbb{R}^{n \times 1}$, and $\mathbb{R}_{+}^{n}$ stands for all non-negative vectors in $\mathbb{R}^{n}$. The sign ' $\geq(>)$ ' represents the entry wise inequality of matrices or vectors. For $A \in \mathbb{R}^{m \times n}$, the aim of this paper is to study various geometric properties of its semipositive cone $K_{A}=\left\{x \in \mathbb{R}_{+}^{n}: A x \in \mathbb{R}_{+}^{m}\right\}$. If the interior of $K_{A}$ is non-empty, then $A$ is a semipositive matrix. The geometric properties of $K_{A}$ generate an interest to review the class of semipositive matrices and their applications.

For an $n \times n$ real matrix $A$, in [15] author proved that $K_{A}$ is a closed, pointed and solid cone in $\mathbb{R}^{n}$. In paricular, author provides a precise structure of the cone $K_{A}$ and shows that $K_{A}$ is a proper polyhedral cone in $\mathbb{R}^{n}$. Further the result has been proved for non-square matrix $A$ in [13]. They also studied the possible inclusion of semipositive cone $K_{A}$ into other $K_{B}$, for any two semipositive matrices $A$ and $B$. In this paper, for any $m \times n$ real matrix $A$ the cone $S_{A}=\left\{x \in \mathbb{R}^{n}: A x \in \mathbb{R}_{+}^{m}\right\}$ containing $K_{A}$, is considered and various properties of the cone parallel to that of $K_{A}$ are studied. Furthermore, given a cone $K$ with specific properties, an attempt is made to classify the matrices $A$ for which either $K=S_{A}$, or $K_{A}$. Again it is known that any two proper cones $K_{1} \subseteq \mathbb{R}^{m}$ and $K_{2} \subseteq \mathbb{R}^{n}$, generate a proper cone $\pi\left(K_{1}, K_{2}\right)=\left\{A \in \mathbb{R}^{m \times n}: A K_{1} \subseteq K_{2}\right\}$ in $\mathbb{R}^{m \times n}$. If the cones $K_{1}=K_{2}=K$, then the cone $\pi(K, K)=\pi(K)$ is the collection of matrices that leave

*Received by the editors on July 28, 2020. Accepted for publication on September 28. Handling Editor: K.C. Sivakumar. Corresponding Author: Manideepa Saha.

$\dagger$ Department of Mathematics, National Institute of Technology Meghalaya, Shillong, Meghalaya, India (ahisabia@nitm.ac.in, manideepa.saha@nitm.ac.in). 
Electronic Journal of Linear Algebra, ISSN 1081-3810

A publication of the International Linear Algebra Society

Volume 36, pp. 764-772, December 2020.

$K$ invariant. In [12], authors proved that $\pi\left(K_{1}, K_{2}\right)$ is a proper polyhedral cone, if $K_{1}$ and $K_{2}$ both are polyhedral cones.

We now describe the paper as follows: Section 2 narrates the definitions and some preliminary results. Section 3 collects cone theoretical results of $S_{A}$, for some given non-singular matrices. In case $A$ is either SP or MSP matrix, then properties of the semipositive cone $K_{A}$ and its extremals are discussed. Furthermore, the relation between $K_{A}$ and $K_{A^{-1}}$ is established in terms of the number of extremals. For a given polyhedral or simplicial cone $K$, Section 4 is devoted to examine matrices $A$ for which either $S_{A}=K$ or the semipositive cone $K_{A}=K$, and the uniqueness of such matrices. Section 5 contains the properties of the cone $\pi\left(K_{A}\right)$ for given minimally semipositive matrix $A$. Finally, the results are summarized in Section 6.

2. Notations and preliminaries. Throughout this paper, we consider real matrices. We refer a matrix $A \in \mathbb{R}^{m \times n}$ as non-negative (positive) if all its entries are non-negative (positive) and in that case, we write $A \geq(>) 0$. Similarly, we denote a non-negative (positive) vector as $x \geq(>) 0$, if all its entries are non-negative (positive). The $i$-th column vector of the identity matrix is denoted by $e_{i}$ with size determined by the context.

An $m \times n$ matrix $A$ is called a semipositive (SP) matrix if there exists a vector $x \geq 0$ such that $A x>0$. As $x \rightarrow A x$ is a continuous mapping, it is equivalent to say that $A$ is $\mathrm{SP}$ if there exists a vector $x>0$ such that $A x>0$. The vector $x$ is referred to as a semipositivity vector of $A$. The set of all SP matrices has two disjoint subclasses, namely minimally semipositive (MSP) matrices and redundantly semipositive (RSP) matrices. For a semipositive matrix $A$ if none of the column deleted submatrix of $A$ is semipositive, then it is known as MSP matrix, otherwise it is called as RSP matrix. We refer to [7, 17, 8, 4, 3] for some basic properties of SP, MSP and RSP matrices.

Some preliminary facts about SP matrices are listed below:

THEOREM 2.1. [7, 8, 17] If $A \in \mathbb{R}^{m \times n}$ is an SP matrix, following results hold:

(i) If $n \geq 2$ and $A$ has a positive (negative) column, then $A$ is $R S P$.

(ii) If $A$ is of full column rank, then the pseudo-inverse $A^{\dagger}$ of $A$ is $S P$.

(iii) If $A$ is $M S P$, then $\operatorname{rank}(A)=n$.

(iv) Square MSP matrices are inverse non-negative matrices and non-square matrices are those matrices having a non-negative left inverse.

TheOrem 2.2. [17] Let $A \in \mathbb{R}^{n \times n}$. Then $A$ is $M S P$ if and only if

$$
A x \geq 0 \Rightarrow x \geq 0
$$

We now discuss some geometric properties, which will be required in the succeeding sections.

Definition 2.3. [1, 2] For any subset $S$ of $\mathbb{R}^{n}$, the set generated by $S$ consists of all finite non-negative linear combinations of elements of $S$, and we denote the set by $S^{G}$. A set $K \in \mathbb{R}^{n}$ is said to be a cone if $K=S^{G}$ for some subset $S$. A subcone of a cone $K$ is any cone contained in $K$.

A cone $K \in \mathbb{R}^{n}$ is called polyhedral cone if there exists a finite set $S$ such that $K=S^{G}$. Equivalently, polyhedral cones are the image of the nonnegative orthant under a linear mapping. A set is called convex, if it contains the line segment joining any two of its points. 
A convex cone $K$ in $\mathbb{R}^{n}$ is called

(i) pointed if $K \cap(-K)=\{0\}$,

(ii) solid if $K$ has non-empty interior,

(iii) reproducing if $K-K=\mathbb{R}^{n}$.

A closed, pointed and solid convex cone is called a proper cone. The dual of a cone $K \in \mathbb{R}^{n}$ is denoted by $K^{*}$ and is defined as

$$
K^{*}=\left\{x \in \mathbb{R}^{n}: x^{T} y \geq 0 \text { for all } y \in K\right\}
$$

A proper cone $K$ always generates a partial order in $\mathbb{R}^{n}$ via $y \stackrel{K}{\leq} x$ if and only if $x-y \in K$. A vector $x$ in $\mathbb{R}^{n}$ is an extremal of $K$ in $\mathbb{R}^{n}$ if $0 \stackrel{K}{\leq} y \stackrel{K}{\leq} x$ implies that $y=\alpha x$, for some $\alpha \geq 0$. If $x$ is an extremal vector of cone $K$, then $\{x\}^{G}$ is called an extremal ray of $K$, and in that case it can be verified that $x \in \partial K$, the boundary of $K$. If a cone $K$ has exactly $n$ extremals, then the cone $K$ is called a simplicial cone. Equivalently, $K$ is a simplicial cone if $K=B \mathbb{R}_{+}^{n}$, for some non-singular matrix $B$ of size $n$.

Let $K_{1} \in \mathbb{R}^{n}$ and $K_{2} \in \mathbb{R}^{m}$ be two proper cones. Then the set $\pi\left(K_{1}, K_{2}\right)$ in $\mathbb{R}^{m \times n}$ is defined as

$$
\pi\left(K_{1}, K_{2}\right)=\left\{A \in \mathbb{R}^{m \times n}: A\left(K_{1}\right) \subseteq K_{2}\right\}
$$

It is well known that $\pi\left(K_{1}, K_{2}\right)$ is a proper cone in $\mathbb{R}^{m \times n}$ (see [1]). In case, $m=n$ and $K_{1}=K_{2}=K$, for simplicity we write $\pi(K)$ for $\pi(K, K)$.

For a non-zero vector $a \in \mathbb{R}^{n}$ and $b \in \mathbb{R}$, we define

(a) a hyperplane as the set $\left\{x \in \mathbb{R}^{n}: a^{T} x=b\right\}$,

(b) a (closed) halfspace as the set $\left\{x \in \mathbb{R}^{n}: a^{T} x \leq b\right\}$.

Note that a hyperplane divides the Euclidean space $\mathbb{R}^{n}$ into two halfspaces.

We now state a few basic results related to cones, which are used in the following sections.

THEOREM 2.4. [1] Let $K$ be a non-empty set in $\mathbb{R}^{n}$. Then following results hold.

(i) $K$ is a polyhedral cone if and only if it is the intersection of finitely many closed half spaces, each containing the origin on its boundary.

(ii) If $K$ is a polyhedral cone, then $K$ is a closed convex cone.

(iii) A nonempty subset $K$ of $\mathbb{R}^{n}$ is a polyhedral cone if and only if its dual $K^{*}$ is a polyhedral cone.

THEOREM 2.5. [1, 16] A proper cone is generated by its extremals.

TheOREM 2.6. [1] Let $K_{1}$ and $K_{2}$ be proper cones, in $\mathbb{R}^{n}$ and $\mathbb{R}^{m}$, respectively. Then

$$
\pi\left(K_{1}, K_{2}\right)=\left\{A \in \mathbb{R}^{m \times n}: A\left(K_{1} \backslash\{0\}\right) \subseteq \operatorname{int} K_{2}\right\}
$$

Definition 2.7. $[13,15]$ For any $A \in \mathbb{R}^{m \times n}$, the semipositive cone of $A$ is denoted by $K_{A}$, and is defined as

$$
K_{A}=\left\{x \in \mathbb{R}_{+}^{n}: A x \in \mathbb{R}_{+}^{m}\right\} .
$$

Note that $K_{A}$ is a cone and if $\operatorname{int}\left(K_{A}\right) \neq \emptyset$, then $A$ is a SP matrix and any vector $\operatorname{in} \operatorname{int}\left(K_{A}\right)$ is a semipositivity vector of $A$. 
A few properties of semipositive cones, mainly due to [13], are given below:

TheOREM 2.8. $[13,15]$ If $A \in \mathbb{R}^{m \times n}$, then $K_{A}$ is proper polyhedral cone.

TheOrem 2.9. [13] If $A \in \mathbb{R}^{n \times n}$ is invertible, then $K_{A}=\mathbb{R}_{+}^{n} \cap A^{-1}\left(\mathbb{R}_{+}^{n}\right)$ and $A\left(K_{A}\right)=K_{A^{-1}}$.

Definition 2.10. For any $A \in \mathbb{R}^{m \times n}$, define

$$
S_{A}=\left\{x \in \mathbb{R}^{n}: A x \in \mathbb{R}_{+}^{m}\right\} .
$$

Note that $S_{A}$ is also a cone in $\mathbb{R}^{n}$, which is not necessarily a proper cone and $K_{A}=S_{A} \cap \mathbb{R}_{+}^{n}$.

3. Properties of $S_{A}$ and semipositive cone $K_{A}$. In [13], it is proved that $K_{A}$ is a polyhedral cone, for a given matrix $A$. In this section, we study various properties of the cones $S_{A}$ and $K_{A}$ in $\mathbb{R}^{n}$, for a given non-singular matrix $A \in \mathbb{R}^{n \times n}$, and analyze their relation if $A$ is either SP or MSP matrix. We also discuss the extremals of these cones in relevance to the inverse of $A$.

TheOREM 3.1. For an invertible matrix $A \in \mathbb{R}^{n \times n}, S_{A}$ is a proper polyhedral cone.

Proof. As any $z \in \mathbb{R}_{+}^{n}$ can be written as $z=A\left(A^{-1} z\right) \geq 0$, so $A\left(S_{A}\right)=\mathbb{R}_{+}^{n}$, that is, $S_{A}=A^{-1}\left(\mathbb{R}_{+}^{n}\right)$, and hence, $S_{A}$ is a polyhebdral cone, being image of nonnegative orthant under the linear map $A^{-1}$.

Again $A$ is invertible implies $S_{A}$ is pointed. The interior of $S_{A}$ is

$$
\operatorname{int}\left(S_{A}\right)=\left\{x \in \mathbb{R}^{n}: A x>0\right\} .
$$

As $A^{-1} y \in \operatorname{int}\left(S_{A}\right)$ for any positive vector $y$, so $S_{A}$ is a solid cone. Thus $S_{A}$ is a proper polyhedral cone.

TheOREm 3.2. For an invertible matrix $A \in \mathbb{R}^{n \times n}$, the extremals of the simplicial cone $S_{A}$ have the form $\alpha_{i} A^{-1} e_{i}$ for some $\alpha_{i}>0, i=1,2, \ldots, n$.

Proof. From the proof of Theorem 3.1, we have that $A\left(S_{A}\right)=\mathbb{R}_{+}^{n}$. Since $A$ is an $n \times n$ invertible matrix, $S_{A}$ is a simplicial cone.

Choose $n$-distinct vectors $x_{1}, x_{2}, \ldots, x_{n}$ in $S_{A}$, whose image under $A$ lies in different axes of $\mathbb{R}_{+}^{n}$. Theorem 3.1 assures the existence of such vectors. Without loss of generality, we assume that for each $i, A x_{i}$ lies in the $i$ th-axis, that is, $A x_{i}=\alpha_{i} e_{i}$, for some positive scalar $\alpha_{i}$.

Let $0 \stackrel{S_{A}}{\leq} y \stackrel{S_{A}}{\leq} x_{i}$, so that $y \in S_{A}$ and $x_{i}-y \in S_{A}$. As $A\left(S_{A}\right)=\mathbb{R}_{+}^{n}, 0 \leq A y \leq A x_{i}=\alpha_{i} e_{i}$. Hence, $A y=\beta e_{i}=\gamma_{i} A x_{i}$ and $\gamma_{i}=\frac{\beta}{\alpha_{i}} \geq 0$. Since $A$ is invertible, $y=\gamma_{i} x_{i}$. Therefore, $x_{i}$ is an extremal of $S_{A}$.

We now prove that $x_{1}, x_{2}, \ldots, x_{n}$ are the only extremals of $S_{A}$. Let $x \in S_{A}$ for which $A x$ has at least two positive entries. Write $A x=b_{n_{1}} e_{n_{1}}+\cdots+b_{n_{k}} e_{n_{k}}$, where $k \geq 2$ and each $b_{n_{j}}(1 \leq j \leq k)$ is a positive scalar with $1 \leq n_{j} \leq n$. Choose $y$ such that $A y=b_{i} e_{i}$. Then $0 \stackrel{S_{A}}{\leq} y \stackrel{S_{A}}{\leq} x$ but $y$ is not expressible as a positive scalar multiple of $x$. Thus, $x$ is not an extremal of $S_{A}$. Thus, the result follows.

The following example illustrates the fact that the semipositive cone $K_{A}$ may not be simplicial, for an invertible matrix $A$.

ExAmPLE 3.3. Consider the invertible matrix

$$
A=\left[\begin{array}{ccc}
0 & 0 & 1 \\
\frac{1}{2} & \frac{1}{5} & -1 \\
0 & 1 & 0
\end{array}\right]
$$


It can be verified that $\left[1,0, \frac{1}{2}\right]^{T},[1,0,0]^{T},[0,1,0]^{T}$ and $\left[0,1, \frac{1}{5}\right]^{T}$ are extremals of $K_{A}$, and so $K_{A}$ is not a simplicial cone.

Theorem 3.4. For an invertible SP matrix $A \in \mathbb{R}^{n \times n}$, if $x$ is an extremal of $K_{A}$, then $A x$ is an extremal of $K_{A^{-1}}$. Moreover, if $p$ is an extremal of $K_{A^{-1}}$, then $A^{-1} p$ is an extremal of $K_{A}$.

Proof. From Theorem 2.9, we have $A\left(K_{A}\right)=K_{A^{-1}}=K$ (say), and hence, $A x \in K$. Let $0 \stackrel{K}{\leq} y \stackrel{K}{\leq} A x$. Then $y \in K$, and so we can choose $z \in K_{A}$ with $y=A z$. Again $A x-y \in K$ implies that $A(x-z) \in K$, that is, $x-z \in K_{A}$. Thus, $0 \stackrel{K_{A}}{\leq} z \stackrel{K_{A}}{\leq} x$, and therefore, $z=c x$ for some $c>0$, or equivalently, $y=c A x$. Hence $A x$ is an extremal of $K=K_{A^{-1}}$.

Let $0 \stackrel{K_{A}}{\leq} q \stackrel{K_{A}}{\leq} A^{-1} p$. Then $A^{-1} p-q \in K_{A}$, that is, $p-A q \in K$, so that $0 \stackrel{K}{\leq} A q \stackrel{K}{\leq} p$. Therefore, $p=\alpha A q$ for some $\alpha>0$, or equivalently, $q=\frac{1}{\alpha} A^{-1} p$. Hence $A^{-1} p$ is an extremal of $K_{A}$.

Corollary 3.5. For an invertible SP matrix $A$, the number of extremals of $K_{A}$ and $K_{A^{-1}}$ are same.

In Example 3.3, we observe that $K_{A}$ may not be a simplicial cone for an invertible matrix $A$. We consider the question as to whether there is a subclass of invertible matrices, for which $K_{A}$ is a simplicial cone. This question is answered in the next theorem.

Theorem 3.6. Any matrix $A \in \mathbb{R}^{n \times n}$ is minimally semipositive if and only if $K_{A}=S_{A}$ and $K_{A}$ is a simplicial cone.

Proof. Let $A$ be a minimally semipositive matrix. Then $A^{-1} \geq 0$, and hence, for $y \in S_{A}, y=A^{-1}(A y) \geq$ 0 , that is, $S_{A}=K_{A}$. So $K_{A}$ is a simplicial cone by Theorem 3.2.

Conversely, assume that $K_{A}=S_{A}$ is a simplicial cone and so $K_{A}$ is a solid cone. Hence $A$ is a semipositive matrix. Let $x \in \mathbb{R}^{n}$ and $A x \geq 0$. Then $x \in S_{A}=K_{A}$, and thus, $x \geq 0$, which shows that $A$ is minimally semipositive by Theorem 2.2 .

Theorem 3.6 leads to the following conclusion.

Corollary 3.7. Let $A \in \mathbb{R}^{n \times n}$. Then

(i) $A$ is an $S P$ matrix if and only if int $S_{A}$ intersects $\mathbb{R}_{+}^{n}$, and

(ii) $A$ is an MSP matrix if and only if $S_{A}$ lies entirely in $\mathbb{R}_{+}^{n}$.

COROLlary 3.8. For a square RSP matrix A, there exists a vector $x$ with at least one negative entry such that $A x>0$.

Remark 3.9. In Theorem 3.6, the condition $K_{A}=S_{A}$ cannot be dropped. Take

$$
A=\left[\begin{array}{lll}
0 & 3 & -1 \\
0 & 1 & -1 \\
0 & 2 & -1
\end{array}\right] .
$$

Note that $K_{A} \neq S_{A}$ as $[1,1,-1]^{T} \in S_{A}$. It can be checked that $K_{A}$ is simplicial with extremals $[1,0,0]^{T}$, $[0,1,0]^{T}$ and $[0,1,1]^{T}$, but $A$ is not a minimally semipositive matrix being a singular matrix.

In Theorem 3.6, we notice that $K_{A}$ is a simplicial cone if $A$ is an MSP matrix, and we now find out the extremals $K_{A}$.

TheOREM 3.10. For a square minimally semipositive matrix $A$, extremals of $K_{A}$ are the columns of $A^{-1}$. 
Proof. As $A$ is MSP, so $A^{-1} \geq 0$ by Theorem 2.1, and hence, $K_{A^{-1}}=\mathbb{R}_{+}^{n}$, which implies that $\left\{e_{1}, e_{2}, \ldots, e_{n}\right\}$ is the set of the extremals of $K_{A^{-1}}$. Since $A\left(K_{A}\right)=K_{A^{-1}}$, so by Theorem $3.4,\left\{A^{-1} e_{1}, A^{-1} e_{2}\right.$, $\left.\ldots, A^{-1} e_{n}\right\}$ is the set of extremals of $K_{A}$, and thus, the result follows.

4. Simplicial cones and related matrices. In Section 3, we studied the geometric structure of $S_{A}$ and $K_{A}$ for a given matrix $A$ with specific property. In this section, we explore the possibilities if the converse is also true. More precisely, given a simplicial or polyhedral cone $K$, our aim is to find out the classes of matrices $A$ for which either $K=S_{A}$ or $K=K_{A}$. We also discuss the uniqueness of such matrices.

In Theorem 3.2, we have seen that $S_{A}$ is a simplicial cone, for invertible matrix $A$. Following theorem gives an affirmative answer if the converse of Theorem 3.2 is true.

THEOREM 4.1. Let $K$ be a simplicial cone in $\mathbb{R}^{n}$. We then have:

(a) There exists an invertible matrix $A$ such that $S_{A}=K$.

(b) If $K$ contains a positive vector, then there exists an invertible $S P$ matrix $A$ such that $K=S_{A}$.

(c) If $K \subseteq \mathbb{R}_{+}^{n}$, then there exists a MSP matrix A such that $K=K_{A}$.

Proof. (a) As $K$ is simplicial, so by definition we can write $K=B \mathbb{R}_{+}^{n}$, where $B$ is invertible and the columns of $B$ are extremals of $K$. Taking $B=A^{-1}$, we get $S_{A}=K$.

(b) Let $p$ be a positive vector in $K$. By Part (a), there exists an invertible matrix $A$ such that $S_{A}=K$. It suffices to show that $A$ is $\mathrm{SP}$.

Since $p$ is positive, we can always choose an open ball $B(p, \epsilon)$ contains only positive vectors. As $S_{A}$ is a closed, so there exists $q$ such that $q \in B(p, \epsilon) \cap$ int $S_{A}$. So $q>0$ and $A q>0$.

(c) Choose an invertible SP matrix $A$ such that $S_{A}=K$, which is possible by (b). As $K$ lies in the positive orthant, so $K=S_{A}=K_{A}$. We only need to show that $A$ is an MSP matrix.

Let $y \in \mathbb{R}^{n}$ and $A y \geq 0$. Then $y \in S_{A}=K$, and hence, by our assumption, $y \geq 0$. So, $A$ is an MSP matrix by Theorem 2.2 .

In Theorem 4.1, we have seen that any simplicial cone $K$ must have the form $K=S_{A}$, for some invertible matrix $A$. Following theorem provides the uniqueness of such matrix $A$ up to post-multiplying by a positive diagonal matrix.

THEOREM 4.2. Let $K$ be a simplicial cone in $\mathbb{R}^{n}$, and let $A$ and $B$ are two invertible matrices such that $K=S_{A}=S_{B}$. Then there exists a positive diagonal matrix $D$ such that $A=B D$.

Proof. Let $\left\{x_{1}, x_{2}, \ldots, x_{n}\right\}$ be the extremals of $K$. As $K=S_{A}=S_{B}$, from Theorem 3.2, we have positive scalar $z_{i}$ and $w_{j}$ such that

$$
x_{i}=z_{i} A^{-1} e_{i}=w_{j} B^{-1} e_{j}, \quad i, j=1,2, \ldots, n,
$$

or

$$
z_{i} B e_{i}=w_{j} A e_{j}, \quad i, j=1,2, \ldots, n
$$

Choose $D_{1}=\operatorname{diag}\left(z_{1}, z_{2}, \ldots, z_{n}\right)$ and $D_{2}=\operatorname{diag}\left(w_{1}, w_{2}, \ldots, w_{n}\right)$. The equation (4.1), can be written $B D_{1} P=A D_{2}$, for some permutation matrix $P$. As $D_{1}$ and $D_{2}$ are positive diagonal matrices, so conclusion follows by considering the positive diagonal matrix $D=D_{1} P D_{2}^{-1}$. 
TheOREM 4.3. For every SP matrix $A$, there exists a MSP matrix $B$ such that $K_{B} \subseteq K_{A}$.

Proof. Let $A$ be any SP matrix and $p>0$ be such that $A p>0$. Note that $p \in \operatorname{int} K_{A}$ and so we can find $\epsilon>0$ such that $B(p, \epsilon) \subseteq \operatorname{int} K_{A}$. Choose $n$ linearly independent vectors $y_{1}, y_{2}, \ldots, y_{n}$ (say) in $B(p, \epsilon)$. Set $\widetilde{A}^{-1}=\left[y_{1}, y_{2}, \ldots, y_{n}\right]$ so that by Theorem $3.2, S_{\widetilde{A}}$ is a simplicial cone with $\alpha_{1} y_{1}, \alpha_{2} y_{2}, \ldots, \alpha_{n} y_{n}$ as its extremals with $\alpha_{i}>0$, for all $i$. Hence by Theorem 4.1(c) there exists a MSP matrix $B$ such that $K_{B}=S_{\widetilde{A}}=\left\{\alpha_{1} y_{1}, \alpha_{2} y_{2}, \ldots, \alpha_{n} y_{n}\right\}^{G}$. As $B(p, \epsilon) \subseteq \operatorname{int} K_{A}$, so $K_{B} \subseteq K_{A}$.

The above theorem together with Theorem 3.6 reveals that every solid semipositive cone contains a simplicial cone.

THEOREM 4.4. Let $K$ be any proper polyhedral cone in $\mathbb{R}^{n}$ such that one of its extremals lie in the positive orthant. Then there exists a minimally semipositive matrix $A$ such that $K_{A}$ is a subcone of $K$.

Proof. Let $x \in \operatorname{int} \mathbb{R}_{+}^{n}$ be an extremal of $K$. Choose an open ball $B(x, \epsilon) \subseteq \operatorname{int} \mathbb{R}_{+}^{n}$. As $x \in \partial K$, $B(x, \epsilon) \cap \operatorname{int} K \neq \phi$. Hence,

$$
\operatorname{int} \mathbb{R}_{+}^{n} \cap \operatorname{int} K \neq \phi .
$$

Now let $V=K \cap \mathbb{R}_{+}^{n}$. Since $K$ and $\mathbb{R}_{+}^{n}$ both are proper polyhedral cone and int $V=\operatorname{int} \mathbb{R}_{+}^{n} \cap$ int $K \neq \phi, V$ is also a proper polyhedral cone. Hence $V=\left\{x_{1}, \ldots, x_{k}\right\}^{G}$. Since $V-V=\mathbb{R}^{n}$, so $\mathbb{R}^{n}=\operatorname{span}\left\{x_{1}, \ldots, x_{k}\right\}$. So, $k \geq n$ and we choose $n$-linearly independent extremals, say $\left\{x_{1}, \ldots, x_{n}\right\}$. Consider the simplicial subcone $\widetilde{K}=\left\{x_{1}, \ldots, x_{n}\right\}^{G}$ of $V$ and hence of $K$. Hence by Theorem 4.1 there exists a minimally semipositive matrix $A$ such that $K_{A}=\widetilde{K}$.

From the above theorem, we can conclude that every polyhedral cone contains a simplicial cone.

5. Matrices leaving a cone invariant: $\pi(K)$. In [12], authors proved that for any two proper cones $K_{1}$ and $K_{2}$ in $\mathbb{R}^{n}$ and $\mathbb{R}^{m}$, respectively, $\pi\left(K_{1}, K_{2}\right)$ is a proper cone. Also $\pi\left(K_{1}, K_{2}\right)$ is a proper polyhedral cone if so are $K_{1}$ and $K_{2}$. In this section, we discuss the geometric structure of $\pi\left(K_{A}\right)$, for a given MSP matrix $A$.

THEOREM 5.1. If $A$ is square MSP matrix, then $\pi\left(K_{A}\right)$ is a simplicial cone.

Proof. Since $A$ is a MSP matrix, $K_{A}$ is a simplicial cone with $A^{-1} e_{1}, \ldots, A^{-1} e_{n}$ as extremals of $K_{A}$. For $i=1,2, \ldots, n$, set $\tilde{a_{i}}=A^{-1} e_{i} \geq 0$, as $A$ is MSP. If $b_{i}^{T}$ denotes the $i$ th row of $A$, then

$$
b_{i}^{T} \tilde{a_{j}}=\delta_{i j} .
$$

For $p, q \in\{1,2, \ldots, n\}$, we define $B_{p q}$ (as in Lemma 5 of [12]) by,

$$
B_{p q}=\tilde{a_{p}} b_{q}^{T} \text {. }
$$

Note that $\mathcal{B}=\left\{B_{p q}: 1 \leq p, q \leq n\right\}$ forms a basis of $\mathbb{R}^{n \times n}$. Let $x \in K_{A}$ so that $x \geq 0$ and $A x \geq 0$. Now

$$
A B_{p q} x=A \tilde{a_{p}} b_{q}^{T} x=\left(b_{q}^{T} x\right) e_{p} \geq 0
$$

Hence $B_{p q} \in \pi\left(S_{A}\right)=\pi\left(K_{A}\right)$, by Theorem 3.6. We now prove that $\pi\left(K_{A}\right)$ is simplicial by showing that the extremals of $\pi\left(K_{A}\right)$ are the elements of $\mathcal{B}$.

Let $Y \in \pi\left(K_{A}\right)$. Choose scalars $d_{r l}$ such that

$$
Y=\sum_{r, l=1}^{n} d_{r l} B_{r l} .
$$


Now for any $x \in K_{A}$, we have $Y x \in K_{A}$ so that $Y x \geq 0$ and $A(Y x) \geq 0$. Then

$$
b_{i}^{T} Y x \geq 0 \quad \text { for } i=1,2, \ldots, n
$$

Note that $\tilde{a_{j}} \in K_{A}$. Taking $x=\tilde{a_{j}}$ in equation (5.4) we obtain $b_{i}^{T} Y \tilde{a_{j}} \geq 0$, for $i, j=1,2, \ldots, n$. Hence equation (5.3) implies that

$$
\sum_{r, l=1}^{n} d_{r l} b_{i}^{T} B_{r l} \tilde{a}_{j} \geq 0
$$

or

$$
\sum_{r, l=1}^{n} d_{r l} b_{i}^{T} \tilde{a_{r}} b_{l}^{T} \tilde{a_{j}} \geq 0
$$

or

$$
d_{i j} \geq 0
$$

So, from (5.3) we conclude that any $Y \in \pi\left(K_{A}\right)$, can be written as non-negative linear combination of elements of $\mathcal{B}$.

For simplicity, write $K=\pi\left(K_{A}\right)$. Let $0 \stackrel{K}{\leq} Y \stackrel{K}{\leq} B_{p q}$. Then

$$
Y=\sum_{r, l=1}^{n} d_{r l} B_{r l} \quad \text { and } \quad B_{p q}-Y=\sum_{r, l=1}^{n} f_{r l} B_{r l}
$$

for some $d_{r l}, f_{r l} \geq 0$ and $r, l \in\{1,2, \ldots, n\}$. Now $B_{p q}=\sum_{r, l=1}^{n}\left(d_{r l}+f_{r l}\right) B_{r l}$ implies that $d_{r l}=f_{r l}=0$, for $r \neq p$ and $l \neq q$. So $Y=d_{p q} B_{p q}$ and $d_{p q} \geq 0$, or equivalently, we say that elements of $B_{p q}$ are extremals of $\pi\left(K_{A}\right)$. As each element in $\pi\left(K_{A}\right)$ is a non-negative linear combination of the extremals vectors in $\mathcal{B}$, so $\mathcal{B}$ is the set all extremals of $\pi\left(K_{A}\right)$. Therefore $\pi\left(K_{A}\right)$ is simplicial.

TheOrem 5.2. If $A$ is an $S P$ matrix, then any matrix $B \in \operatorname{int} \pi\left(K_{A}\right)$ is also $S P$ and $K_{A} \subseteq K_{B}$.

Proof. Let $u$ be a semipositivity vector of $A$. Note $u \in K_{A} \backslash\{0\}$. As $B \in \operatorname{int} \pi\left(K_{A}\right)$, from Theorem 2.6, we have $B u \in \operatorname{int}\left(K_{A}\right)$, and hence, $B u>0$. This shows that $B$ is SP and $u \in K_{B}$. Hence $K_{A} \subseteq K_{B}$.

6. Conclusion. In this paper, we have proved that the cone $S_{A}$ is a simplicial cone, for a given nonsingular matrix $A$. We have also shown that $S_{A}$ is a semipositive cone if $A$ is a minimally semipositive matrix and the extremals of $K_{A}$ are nothing but the columns of $A^{-1}$. Next, for a given simplicial cone $K$, we proved the existence of a nonsingular matrix $A$ for which $S_{A}=K$ and the uniqueness of such a matrix is also discussed. Lastly, for a given matrix $A$, we have considered the cone $\pi\left(K_{A}\right)$ of all matrices that leave the semipositive cone $K_{A}$ invariant. In particular, we have proved that if the matrix $A$ is minimally semipositive matrix, then cone $\pi\left(K_{A}\right)$ must be a simplicial cone.

Acknowledgment. The authors would like to express their gratitude to the anonymous referees for careful reviews and insightful comments, that significantly improved the quality of the paper. 


\section{REFERENCES}

[1] A. Berman and R.J. Plemmons. Nonnegative Matrices in the Mathematical Sciences. Academic Press, New York, 1979.

[2] S. Boyd and L. Vandenberghe. Convex Optimization. Cambridge University Press, Cambridje, 2004.

[3] P.N. Choudhury, R.M. Kannan, and K.C. Sivakumar. New contributions to semipositive and minimally semipositive matrices. Electron. J. Linear Algebra, 34:35-53,2018.

[4] J. Dorsey, T. Gannon, C.R. Johnson, and M. Turnansky. New results about semi-positive matrices. Czechoslovak Math. J., 66:621-632, 2016.

[5] M. Fiedler and V. Pták. Some generalizations of positive definiteness and monotonicity. Numer. Math., 9:163-172, 1966.

[6] R.A. Horn and C.R. Johnson. Matrix Analysis. Cambridge University Press, New York, 1985.

[7] C.R. Johnson, M.K. Kerr, and D.P. Stanford. Semipositivity of matrices. Linear Multilinear Algebra, 37:265-271, 1994.

[8] C.R. Johnson and D.P. Stanford. Qualitative semipositivity. In: R.A. Brualdi, S. Friedland, and V. Klee (editors), Combinatorial and Graph-Theoretical Problems in Linear Algebra, The IMA Volumes in Mathematics and its Applications, Vol 50, Springer, New York, 99-105, 1993.

[9] M.A. Krasnosel'skii. Positive Solutions of Operator Equations. P. Noordhoff, The Netherlands, 1964.

[10] M.G. Krein and M.A. Rutman. Linear operators leaving invariant a cone in a Banach space. Amer. Math. Soc., 1948.

[11] S.W. Park, H.G. Seol, and G. Lee. A characterization of minimal semipositivity of sign pattern matrices. Commun. Korean Math. Soc., 13:465-473, 1998.

[12] H. Schneider and M. Vidyasagar. Cross positive matrices. SIAM J. Numer. Anal., 7:508-519, 1970.

[13] K.C. Sivakumar and M.J. Tsatsomeros. Semipositive matrices and their semipositive cones. Positivity, 22:379-398, 2018.

[14] E. Stiemke. Über positive Lösungen homogener linearer Gleichungen. Math. Ann., 76:340-342, 1915.

[15] M.J. Tsatsomeros. Geometric mapping properties of semipositive Matrices. Linear Algebra Appl., 498:349-359, 2016.

[16] J.S. Vandergraft. Spectral properties of matrices which have invariant cone. SIAM J. Appl. Math., 16(6):1208-1222, 1968.

[17] H.J. Werner. Characterizations of minimal semipositivity. Linear Multilinear Algebra, 37:273-278, 1994. 